\title{
Surface wrinkling for increasing the short circuit current density of thin film solar cells deposited on chemically oxidized silicone surface
}

\author{
Himanshu Ingale, Murali M. Sundaram \\ Department of Mechanical and Materials Engineering \\ University of Cincinnati \\ Cincinnati, Ohio, USA
}

\begin{abstract}
Solar cell technology is one of the most promising sources of clean energy. However, some limitations include low efficiency, high manufacturing cost, and large consumption of material. A novel method of depositing a thin film direct bandgap semiconductor material on lightweight substrate, which would result in higher specific power $(\mathrm{kW} / \mathrm{kg})$, is explored in this work. The efficiency of such solar cells can be further increased by providing a textured surface, resulting in reduced optical losses therefore increasing light trapping. This paper reports a novel method which makes use of a mechanical instability in a soft material (Polydimethylsiloxane) to make wrinkles on its surface by chemically oxidizing the surface using Piranha solution. Further, these wrinkles were arranged in order by applying external force to the soft material before chemical oxidation. Theoretical studies have been carried out and found more than $10 \%$ increase in transmittance and short circuit current if a cadmium telluride (CdTe) solar cell is to be deposited on such substrate.
\end{abstract}

\section{KEYWORDS}

Solar cell texturing, surface wrinkling, light trapping

\section{INTRODUCTION}

Thin film solar cell technology is rapidly gaining attention due to its advantages such as cheap production cost, versatility, flexibility and ease of handling. Conventionally, thin film solar cells are grown on 1-4 $\mathrm{mm}$ thick glass substrates, wherein only a few microns thick layers are needed for the actual solar cell stack. Solar cells with high specific power can be manufactured if glass is substituted with a lightweight substrate, as glass substrate accounts for nearly $90 \%$ of the total weight of the cell. Also, solar cells deposited on flexible and lightweight substrates are advantageous over solar cells deposited on glass for both terrestrial and space applications where high specific power and flexibility are required for curved shaping or rolling. Flexible solar cells give more possibilities for integration into buildings, solar cars and boats, consumer electronics and portable electronics[1]. Cadmium telluride (CdTe) is one of the preferred materials for thin film solar cells, due to its optimum band gap for the efficient photo-conversion and robustness for industrial production[2]. However, most of the thin film solar cells must compromise between achieving complete optical absorption using films that are thicker than the optical absorption length and achieving efficient conversion of the absorbed photons into photocurrent[3]. This results in increased thickness of the active layer of thin film, thus increasing the overall cost of the production. Hence, light trapping is essential to achieve high efficiencies in thin cells[4].

The present research aims to accomplish the aforementioned objective of manufacturing a lightweight flexible polymer substrate with a texturing pattern on it. Buckling instability is taken advantage of in soft material polydimethylsiloxane (PDMS), which consists of repeated units of $-\mathrm{O}-\mathrm{Si}-\left(\mathrm{CH}_{3}\right)_{2}$. PDMS is treated with a piranha solution, a strong acid which is a mixture of sulfuric acid and hydrogen peroxide. This substance causes the formation of an oxidized surface on PDMS that is harder than the regular PDMS substrate $[5,6]$. This leads to the introduction of a bi-layer system, in which the difference in elastic modulus generates compressive stresses and forms surface wrinkles. Furthermore, these wrinkles can be arranged in an orderly fashion under the guidance of external forces before oxidization. 


\section{LITERATURE REVIEW}

When a thin elastic film is coated on top of a pre-strained soft substrate, followed by release of the pre-strain, a mismatch in equilibrium states arises between the two layers, causing a buckling instability in the bilayer system. This leads to formation of various wrinkling patterns that bring the system to a new equilibrium state. When a soft substrate is expanded, some of the external energy is converted into the potential energy within the substrate. When a thin film is coated on the top of the prestrained substrate, followed by release of the strain, a new equilibrium state must be reached due to mismatch of the two equilibrium states between soft substrate and hard thin film. This results in the formation of wrinkles shown schematically in Figure 1, which is the result of minimization of the total elastic energy in the thin layer and the soft substrate.

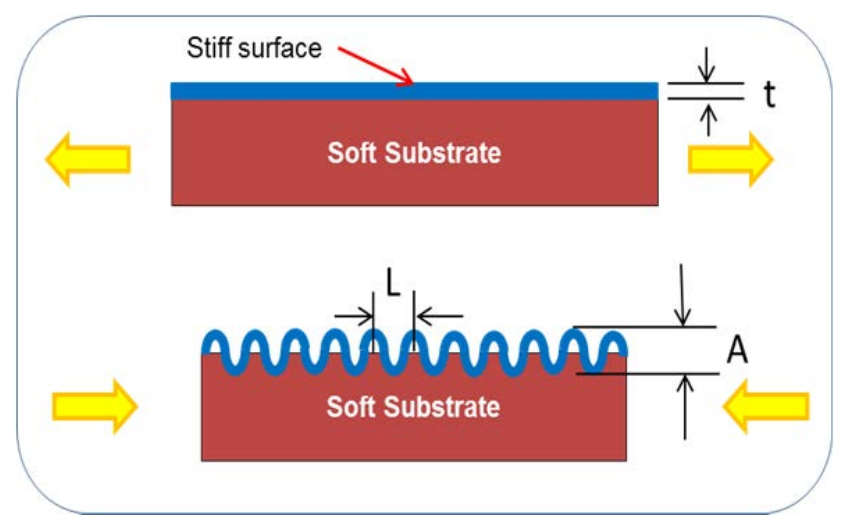

Figure 1. Schematics of wrinkle formation through buckling instability in a bilayer system $(t=$ thickness of hard film, $\mathrm{L}=$ wavelength, $\mathrm{A}=$ amplitude of the wrinkle)

Spontaneous formation of such structures on a solid surface of micrometer to sub-micrometer scale is a potentially more efficient alternative to conventional micromachining techniques. Mechanical buckling instability is one such phenomenon, which has received increased attention for its potential to create ordered wrinkling structures on a micrometer to sub-micrometer scale. Recently, these instabilities have been exploited for assembling colloidal particles [7], stretchable silicon substrates for electronics [8], measuring mechanical properties of thin films [9], protein pre-concentration [10], tunable wetting and tunable open channel microfluidics [11]. Several methods to harness ordered surface wrinkles on soft substrate have been reported so far. These methods include: treating PDMS surface with oxygen plasma [12], UV zone treatment [13] and ion implantation [14].
However, these methods not only require sophisticated equipment, but are also incapable of creating a surface pattern on large region. If spontaneous surface wrinkling can be applied to a large region for creating microstructures, it can be more cost effective than conventional techniques of micro-fabrication. One such method was first proposed that involves treating PDMS surface with the mixture of sulfuric acid and nitric acid [5]. Later, this technique to harness ordered surface wrinkles on PDMS was used for large region surface wrinkling [15].

One example of a simple approach to harness ordered surface wrinkles is to deposit a thin, hard layer on top of a soft, expanded substrate, and then release the strain within the substrate. In this example, wrinkles were fabricated by depositing a thin Au layer on a thermally expanded PDMS film, followed by cooling the substrate to the room temperature [16]. However, since the thin film is not covalently bonded to the soft substrate, it tends to delaminate from the substrate under a large strain. To address this issue, a thin, hard layer can be formed directly on the PDMS surface by oxidizing the top layer. In this study, a chemical method is used to achieve the aforementioned wrinkling on a PDMS surface.

\section{EXPERIMENTATION}

This study involves the use of Piranha solution, which oxidizes the surface of PDMS by forming silanol groups. Piranha solution is a strong acid mixture normally consisting of concentrated sulfuric acid and hydrogen peroxide, commonly used as a cleaning agent for wafers in the soft-lithography process. The chemical reaction between concentrated sulfuric acid with hydrogen peroxide generates hydronium ions, bi-sulphate ions and a reactive atomic oxygen species as shown in equation (1).

$$
\mathrm{H}_{2} \mathrm{SO}_{4}+\mathrm{H}_{2} \mathrm{O}_{2}=\mathrm{H}_{3} \mathrm{O}^{+}+\mathrm{HSO}_{4}^{-}+\mathrm{O}
$$

The reactive atomic oxygen species is the primary agent for the formation of an oxidized PDMS surface, breaking the $\mathrm{Si}-\mathrm{CH}_{3}$ bonds in the substrate to form a silanol group $(\mathrm{Si}-\mathrm{OH})$. The process begins with the destruction of the bonds within a methyl group by the reactive oxygen, forming unstable $\mathrm{CH}_{3} \mathrm{O}^{-}$. This ion is detached from -Si-Obranch and the vacancy in the structure is filled by $\mathrm{OH}^{-}$to form silanol group [6]. The chemistry of the process is shown in figure 2. A schematic of the entire process and experimental setup is shown in figure 3. Experimental materials and methods are described below. 


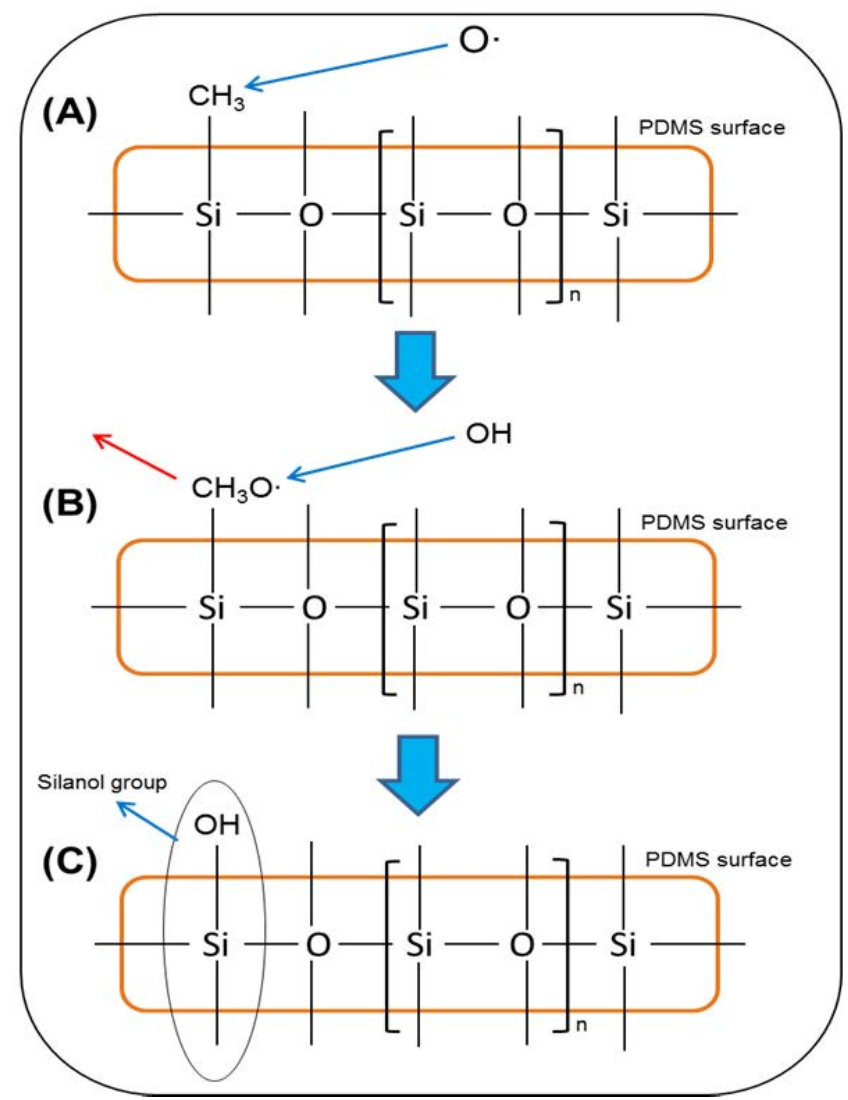

Figure 2. Methyl group in PDMS gets replaced by silanol group. A) Methyl group being attacked by reactive oxygen species from Piranha solution forming unstable $\mathrm{CH}_{3} \mathrm{O}^{-}$. B) Unstable $\mathrm{CH}_{3} \mathrm{O}^{-}$is detached from $-\mathrm{Si}-\mathrm{O}$

branch. C) Left vacancy is taken by $\mathrm{OH}^{-}$to form silanol group.

\section{Materials}

PDMS pre-polymer consisting of siloxane base and curing agent (Sylgard 184 Dow Corning), concentrated sulfuric acid (>96\%) and Hydrogen peroxide (30\%).

\section{Preparation of PDMS substrate}

Two different samples of PDMS were prepared by mixing pre-polymer siloxane base and curing agent in the ratio 10:1 and 10:0.4. The mixture was then poured into a petri dish at a room temperature and allowed to settle for 15 minutes. The PDMS with 10:0.4 siloxane base to curing agent ratio was then divided into two different samples, one of which was cured at $60{ }^{\circ} \mathrm{C}$ for 1 hour and other at $60^{\circ} \mathrm{C}$ for 2 hours. Also, PDMS with 10:1 siloxane base to curing agent ratio was cured at $60{ }^{\circ} \mathrm{C}$ for 1 hour. The obtained PDMS substrate was finally cut into rectangular pieces of $3 \mathrm{~cm} \times 4 \mathrm{~cm}$ each.

\section{Preparation of the acid solution}

An acid solution (40 ml) was prepared by mixing concentrated sulfuric acid with hydrogen peroxide in a $3: 1$ ratio. Hydrogen peroxide was introduced slowly into the concentrated sulfuric acid solution. Since the process involves release of gas and heat, mixing was carried out under fume hood.

\section{Acid treatment of PDMS:}

The obtained rectangular PDMS pieces were observed to be approximately of $2.4 \mathrm{~mm}$ in thickness which were measured by digital calipers of $0.03 \mathrm{~mm}$ precision.

These rectangular pieces were then clamped to a modified bench vise to introduce a strain of $30 \%$ in $\mathrm{x}$ - direction. The effective length of the rectangular PDMS piece held between two jaws of bench vice was measured using the digital calipers. To introduce a strain of $30 \%$ in sample the main screw was rotated by appropriate amount since the pitch of the screw was known to be $2.11 \mathrm{~mm}$. The stretched sample was then treated with Piranha solution for various durations, including 40 seconds and 60 seconds. After being cooled at room temperature in air for 3 minutes, the strain in the sample was slowly released. The sample was then washed with deionized water to remove any trace of the acid and dried for approximately 30 minutes. All the experimental conditions have been summarized in table 1.

\begin{tabular}{|c|c|c|c|c|c|c|}
\hline Material & $\begin{array}{c}\text { Siloxane } \\
\text { base to } \\
\text { cross } \\
\text { linking } \\
\text { agent } \\
\text { ratio }\end{array}$ & $\begin{array}{c}\text { Curing } \\
\text { temperature } \\
\text { in }{ }^{\circ} \mathrm{C}\end{array}$ & $\begin{array}{c}\text { Curing } \\
\text { time } \\
\text { in } \\
\text { minutes }\end{array}$ & $\begin{array}{c}\text { Strain } \\
\text { percentage }\end{array}$ & $\begin{array}{c}\text { Acid } \\
\text { treatment } \\
\text { time in } \\
\text { seconds }\end{array}$ \\
\hline PDMS & $10: 1$ & $60^{\circ} \mathrm{C}$ & 60 & 30 & 40 & 60 \\
\hline PDMS & $10: 0.4$ & $60^{\circ} \mathrm{C}$ & 60 & 30 & 40 & 60 \\
\hline PDMS & $10: 0.4$ & $60^{\circ} \mathrm{C}$ & 120 & 30 & 40 & 60 \\
\hline
\end{tabular}

Table 1. Experimental conditions 


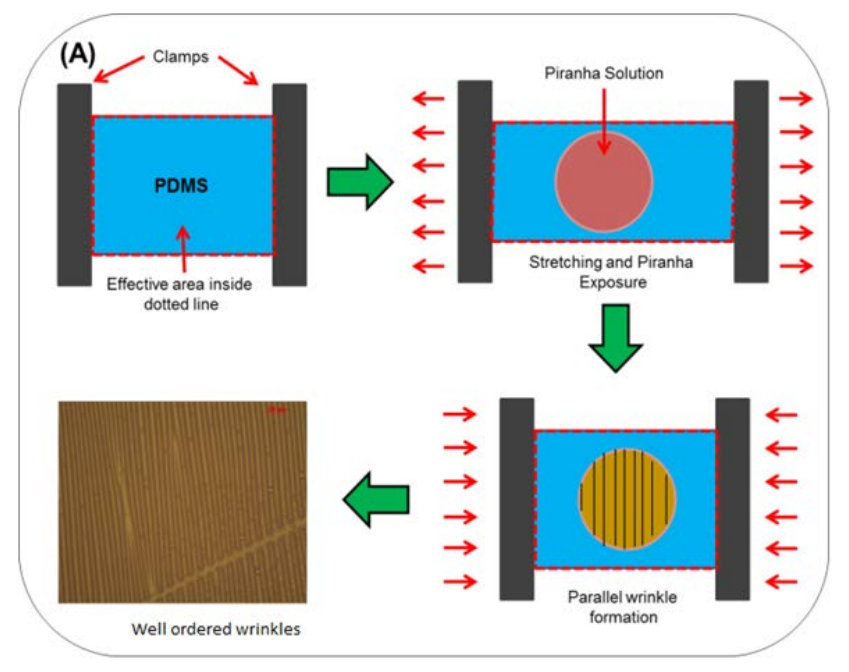

Figure 3. A) Schematic of the experimental procedure.

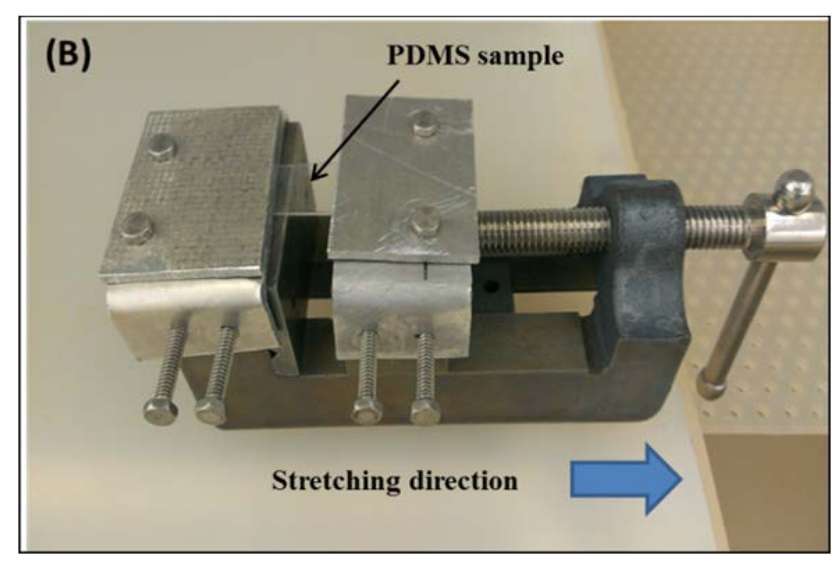

Figure 3. B) Modified bench vise for experiment.

\section{RESULTS AND DISCUSSION}

\section{Effect of acid treatment duration on wrinkle formation:}

It was observed that various samples behave differently to the acid treatment exposure time. In the case of a PDMS sample with 10:0.4 siloxane base to curing agent ratio, which was cured at $60{ }^{\circ} \mathrm{C}$ for 2 hours, an increase in wavelength of the wrinkles was observed. According to linear buckling theory, the wavelength $\lambda$ of the wrinkling pattern depends on the thickness of the thin hard film and other mechanical properties of the substrate[11], as shown by the following relation:

$$
\lambda=2 \pi t\left(\frac{E_{f}}{3 E_{s}}\right)^{\frac{1}{3}}
$$

Where, $E_{f}$ and $E_{s}$ are plain strain modulus of the thin film and compliant substrate, and $t$ is the thickness of the thin hard film. From the equation, it is clear that as the thickness of the thin hard layer increases, the wavelength of the wrinkles will also increase.

Figure 4 shows the increase in the wavelength of the PDMS samples treated for 40 seconds and 60 seconds respectively, which is in accordance with the linear buckling theory.

However, when the PDMS sample with 10:0.4 siloxane base to curing agent ratio was cured for 1 hour at $60{ }^{\circ} \mathrm{C}$ and treated with acid for 40 and 60 seconds respectively, it was observed that most of the treated surface was converted to a silica[6]-like (brittle) material, which prevents the surface wrinkling of the layer, as shown in figure 5 .

Thus, it can also be concluded that the wrinkle formation is also dependent on the curing time of the PDMS as it is found that the acid reacts more rigorously if the curing time is reduced for the same base to cross linking agent ratio material.
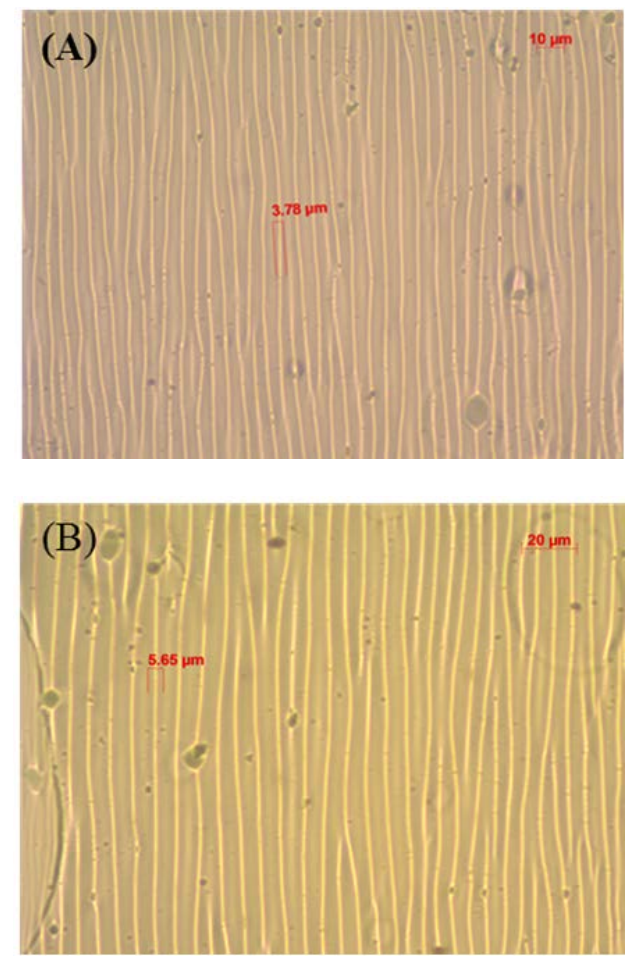

Figure 4. Figure shows the increase in wavelength of the wrinkles with increase in acid treatment time for PDMS with 10:0.4 siloxane base to curing agent ratio treated at $60{ }^{\circ} \mathrm{C}$ for 2 hours, acid treatment time being A) 40 seconds B) 60 seconds respectively. (Microscope - Zeiss Axiovert 40MAT) 


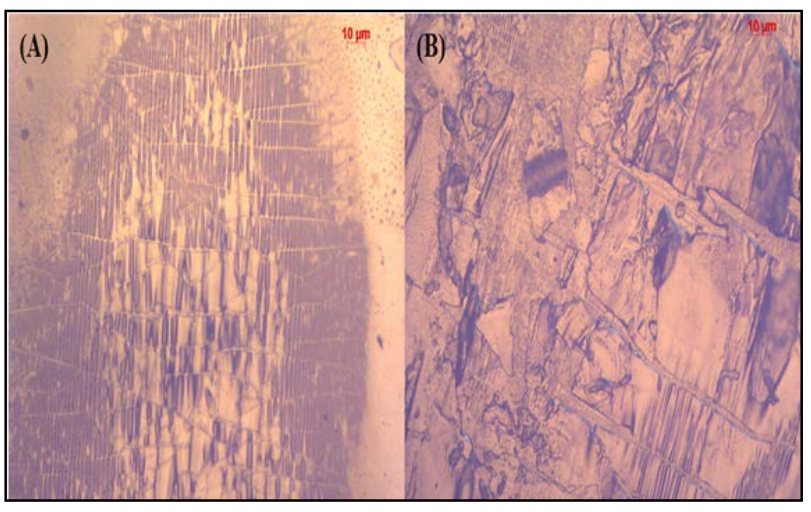

Figure 5. Figure showing formation of silica like layer on PDMS with 10:0.4 siloxane base to curing agent ratio cured at $60{ }^{\circ} \mathrm{C}$ for 1 hour and treated for A) 40 seconds and B) 60 seconds respectively. (Microscope - Axiovert 40MAT)

To determine whether, the variation in the siloxane base to cross linking ratio of the PDMS affects the wrinkle formation, experiments were conducted with PDMS samples of a $10: 1$ siloxane base to curing agent ratio, cured at $60{ }^{\circ} \mathrm{C}$ for 1 hour. It was found that when such PDMS was treated with acid for 40 seconds and 60 seconds respectively, a silica-like layer formed on the treated region due to prolonged exposure to acid, as shown in Figure 6B. This indicates that in order to obtain wrinkles, the treatment time should be reduced. This sample was then treated for 15 seconds, resulting in ordered wrinkle formation on the surface. From these results, it can be concluded that the siloxane base to crosslinking agent ratio also plays a vital role in the wrinkle formation process.

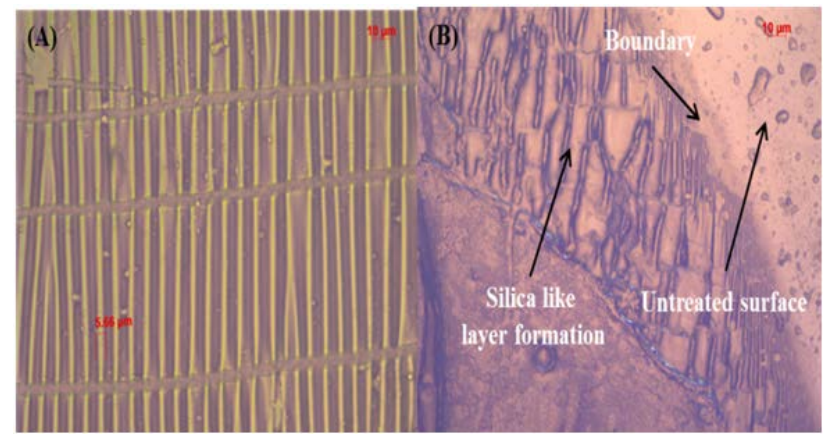

Figure 6. A) Wrinkle formation for 15 second treatment time of PDMS 10:1, cured at $60{ }^{\circ} \mathrm{C}$ for 1 hour. B) Silica like layer is developed on PDMS 10:1, cured at $60{ }^{\circ} \mathrm{C}$ for 1 hour, due to prolonged exposure to acid. (Microscope Axiovert 40MAT)

\section{Formation of ordered wrinkling pattern}

Figure 7, shows an ordered wrinkle pattern on PDMS surface (Siloxane base to cross linking ratio 10:0.4, cured at $60{ }^{\circ} \mathrm{C}$ for 2 hours and having a thickness of approximately $2 \mathrm{~mm}$ ) after being treated with the Piranha solution for 40 seconds. To measure the wavelength and amplitude of the wrinkling pattern, the sample was scanned using KLA-Tencor P-15 Profiler using a force of $10 \mathrm{mg}$ on stylus tip and scanning at $20 \mu \mathrm{m} / \mathrm{sec}$. The profilometer has a scan repeatability of $7.5 \AA$ and reproducibility of $15 \AA$. Figure $7 \mathrm{C}$ shows the 3D profile of the wrinkles on PDMS while figure $7 \mathrm{D}$ shows the 2D profiles of the same wrinkled region. This 2D profile of the wrinkles was used to determine wavelength, amplitude and approximate angle between wrinkles. The wavelength of the wrinkles ranged approximately $10-20$ micron while amplitude ranged approximately $2-6$ micron. The angle between the wrinkles is found to range between $85^{\circ}$ to $130^{\circ}$.
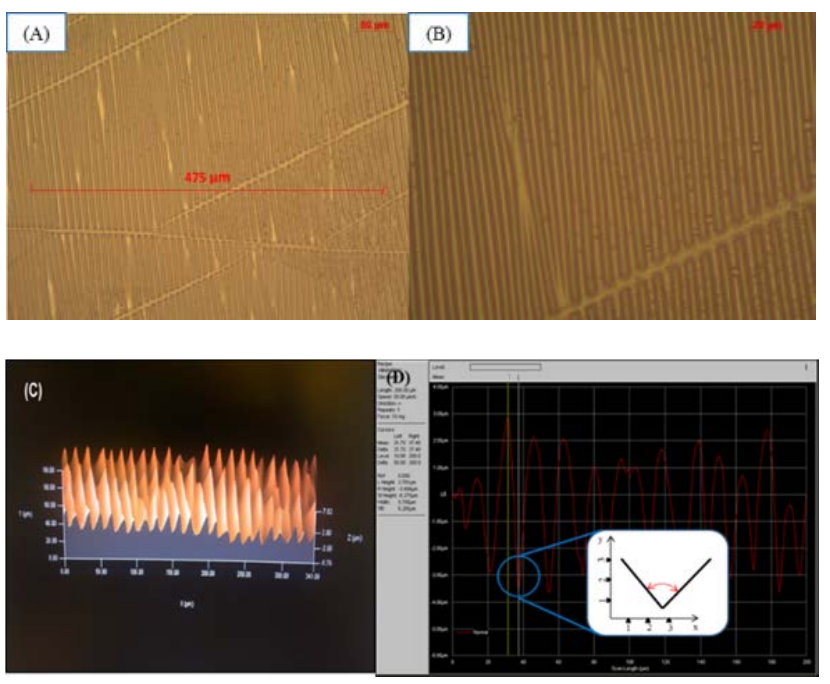

Figure 7. A) Ordered winkles over a large surface area of pdms. B) Zoomed in view of the ordered wrinkles. (Scale $20 \mu \mathrm{m}) \mathrm{C}$ ) $3 \mathrm{D}$ profile of the wrinkles. (X-axis $=0-341$ $\mu \mathrm{m}, \mathrm{Y}$ axis $=0-99 \mu \mathrm{m}, \mathrm{Z}$ axis $=0-7.62 \mu \mathrm{m}) \mathrm{D}) 2 \mathrm{D}$ profile of the wrinkled surface. On the left side of the image, it shows, difference of height between a peak and a valley as $6.275 \mu \mathrm{m}$ and half wavelength as $5.7 \mu \mathrm{m}$. (PDMS: Base to cross linking ratio $10: 0.4$, cured at $60{ }^{\circ} \mathrm{C}$ for 2 hours and thickness of approximately $2 \mathrm{~mm}$ )

However, numbers of cracks were also observed in a direction parallel to $\mathrm{x}$-axis. This is due to Poisson's effect when the substrate is stretched in $\mathrm{x}$-axis direction. Also, there were few round shaped defects which resulted from the burst of micro bubbles trapped in PDMS mixture since PDMS was not degassed before curing. 


\section{Optical losses in thin $\mathrm{CdS} / \mathrm{CdTe}$ solar cells}

To assess the reduction in optical losses of a solar cell deposited on a substrate developed in this work, a theoretical study of the reflection loss, considering CdTe solar cell in superstrate configuration has been carried out. Figure 8 shows the schematic of the superstrate configuration of a CdTe solar cell deposited on glass. Same configuration can be used to deposit CdTe solar cell on a textured PDMS surface developed in this work.
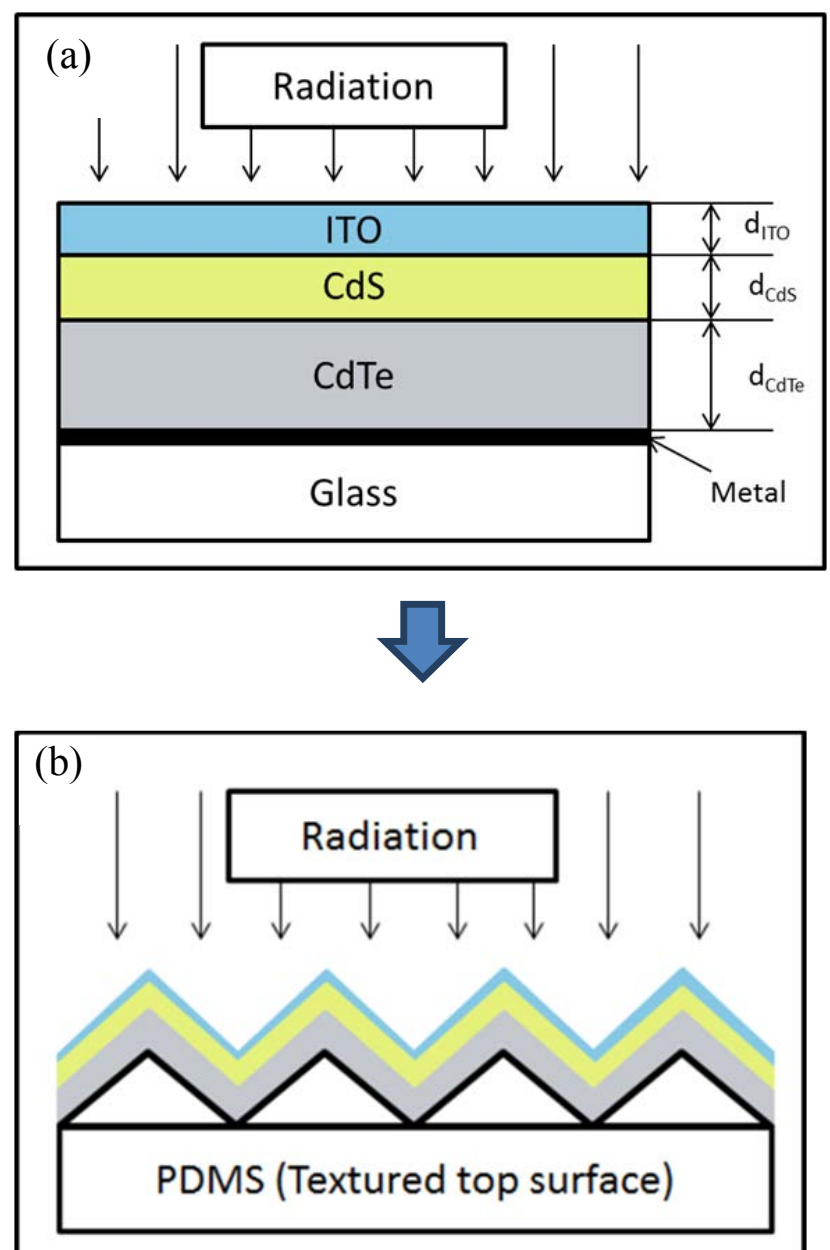

Figure 8. a) Schematic of CdTe solar cell in superstrate configuration. b) Schematic of CdTe solar cell on textured polymer substrate.

CdTe solar cell in superstrate configuration consists of Indium tin oxide) (ITO layer which acts an transparent electrode allowing most of the radiation to pass through it and also collecting the current, the second layer consists of cadmium sulphide (CdS ) which has a bandgap of $2.4 \mathrm{eV}$ [17] due to which it allows most of the low energy photons to pass through it. This CdS layer also provides a junction field for photo-generated minority carriers[18]. The third layer is CdTe which acts as an absorber layer. CdTe has a bandgap of $1.5 \mathrm{eV}$ which is optimum for photovoltaic solar energy conversion. Also, it has high absorption coefficient which allows only a few microns of material to absorb most of the light[19]. The final layer consists of a metal which acts as a back contact for the solar cell and collects current.

Optical losses in CdTe solar cell are due to reflections at the interfaces and absorption in ITO, CdS layers. Calculations have been done based on the optical constants of the materials used in CdTe solar cell (refractive index, extinction coefficient and absorption coefficient). Thus, before the solar radiation reach photo electrically active CdTe absorber layer, it has to pass through ITO and CdS layer where it gets reflected at interfaces air-ITO, ITO-CdS and CdS-CdTe accompanied by absorption in ITO and $\mathrm{CdS}$ layers. According to Fresnel equations, for normal incidence of light, the reflection coefficient from the interface between two mediums is given by

$R=\frac{\left(n_{1}-n_{2}\right)^{2}}{\left(n_{1}+n_{2}\right)^{2}}$

where, $\mathrm{n} 1$ and $\mathrm{n} 2$ are refractive indices of the medium.

However, in case of electrically conductive materials, the refractive index contains an imaginary part known as extinction coefficient which can be represented as

$\mathrm{n}^{*}=\mathrm{n}-\mathrm{ik}$

where,

$\mathrm{n}$ is a refractive index and $\mathrm{k}$ is an extinction coefficient.

The reflection coefficient from the interface of electrically conductive medium is given as[20].

$R=\frac{\left(n_{1}-n_{2}\right)^{2}+\left(k_{1}-k_{2}\right)^{2}}{\left(n_{1}+n_{2}\right)^{2}+\left(k_{1}+k_{2}\right)^{2}}$

Figure 9 shows the refractive and extinction coefficients of ITO, CdS and CdTe with wavelength. 

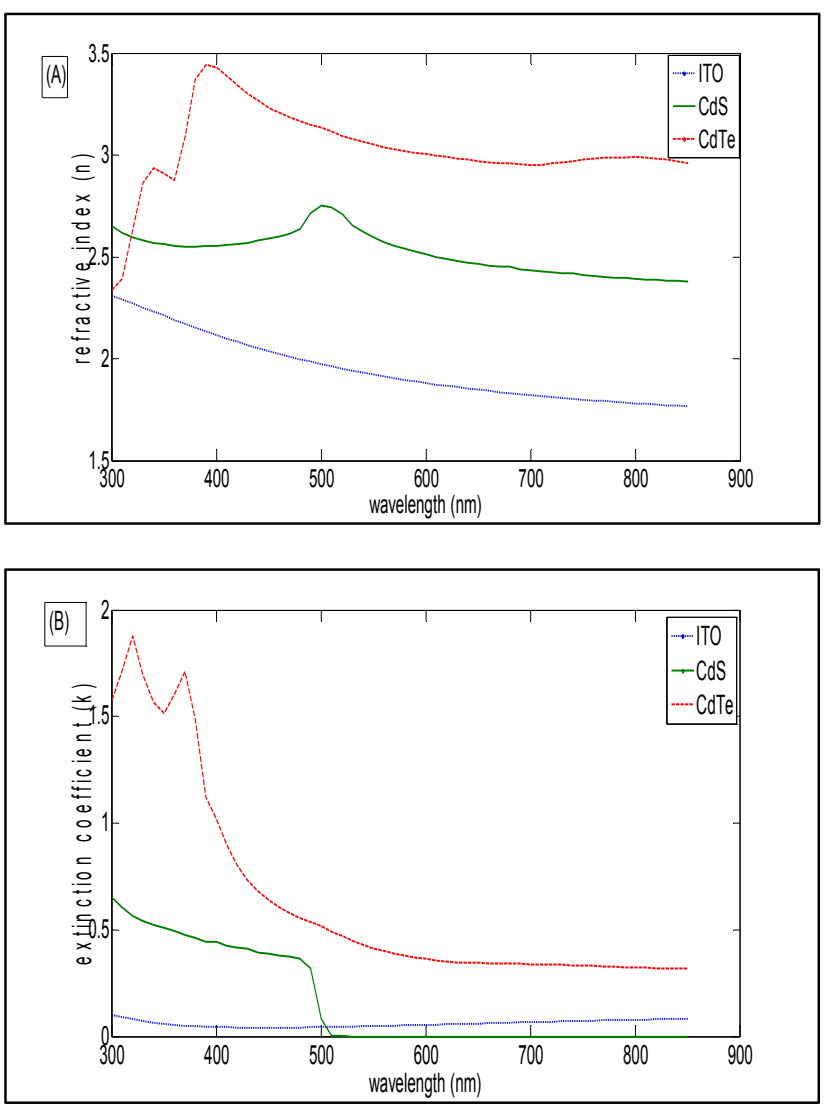

Figure 9. (A) Refractive index and (B) extinction coefficients of ITO, CdS and $\mathrm{CdTe}$ as a function of wavelength

Using the above equations, optical constants[21] of the materials and solar irradiance data for AM1.5G [22], the reflection loss has been calculated for CdTe solar cell in superstrate configuration deposited on a glass substrate and laboratory developed substrate. For calculations, the angle between two wrinkles is taken as $90^{\circ}$ which results in reflection of a light twice from the textured surface[23]. In case of the textured surface, we can write the relative complex index of refraction as the ratio of refractive indices of the two mediums, as[24]

$$
n=\frac{n_{2}}{n_{1}}
$$

$n=\frac{n_{2}-i k_{2}}{n_{1}-i k_{1}}$

This relative index of refraction is further put into the following Fresnel equations for calculating reflective coefficient of the textured surface.

$P_{S}=\frac{u^{2}+v^{2}-2 u \cos \theta+\cos \theta^{2}}{u^{2}+v^{2}+2 u \cos \theta+\cos \theta^{2}}$
$P_{p}=P_{S} * \frac{u^{2}+v^{2}-2 u \sin \theta \tan \theta+\sin \theta^{2} \tan \theta^{2}}{u^{2}+v^{2}+2 u \sin \theta \tan \theta+\sin \theta^{2} \tan \theta^{2}}$

where,

$\mathrm{Ps}=$ s-polarized light, $\mathrm{Pp}=\mathrm{p}$-polarized light. $\theta=$ Angle of incidence.

Thus, we have,

$$
\begin{aligned}
2 u^{2}=n^{2}-k^{2}- & \sin \theta^{2} \\
& +\left(\left(n^{2}-k^{2}-\sin \theta^{2}\right)^{2}+4 n^{2 k^{2}}\right)^{1 / 2} \\
2 v^{2}=k^{2}-n^{2}+ & \sin \theta^{2} \\
& +\left(\left(n^{2}-k^{2}-\sin \theta^{2}\right)^{2}+4 n^{2 k^{2}}\right)^{1 / 2}
\end{aligned}
$$

Using above formulae, Equation (4) for incidence on nontextured surface and equations $(6,7)$ for incidence on textured surface, the transmission percentage into the CdTe absorber layer can be calculated in the following relation, considering the reflection losses at the interfaces between materials and absorption into ITO and $\mathrm{CdS}$ layers:

$$
\begin{gathered}
T(\lambda)=(1-R 12) *(1-R 23) *(1-R 34) * \\
\exp (-\alpha 1 * d 1) * \exp (-\alpha 2 * d 2)
\end{gathered}
$$

where,

$\mathrm{R} 12=$ Reflection coefficient between air-ITO

$\mathrm{R} 23=$ Reflection coefficient between ITO-CdS

R34 = Reflection coefficient between CdS-CdTe

$\alpha 1=$ Absorption coefficient of ITO

$\alpha 2=$ Absorption coefficient of $\mathrm{CdS}$

$\mathrm{d} 1=$ Thickness of ITO $=100 \mathrm{~nm}$

$\mathrm{d} 2=$ Thickness of $\mathrm{CdS}=100 \mathrm{~nm}$

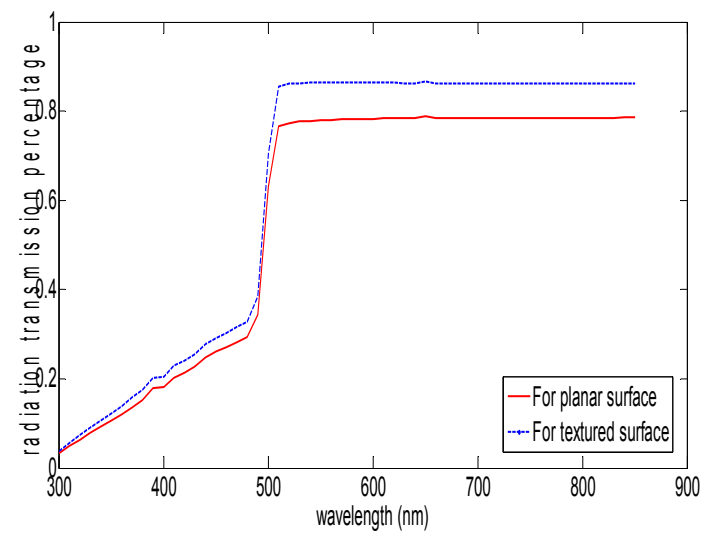

Figure 10. Calculated transmittance into photoelectrically active CdTe layer 
Figure 10 clearly illustrates that a higher amount of light is transmitted into a CdTe solar cell deposited on textured substrate than a conventional solar cell deposited on glass substrate. It is found from the calculations that the transmittance into CdTe solar cell deposited on textured substrate will increase by more than $10 \%$ as compared to planar surface.

\section{Effect of optical losses on short circuit current:}

The short-circuit current is the current through the solar cell when the voltage across the solar cell is zero. The short-circuit current is a result of the generation and collection of light-generated carriers. For an ideal solar cell, the short-circuit current and the light-generated current are identical. Therefore, the short-circuit current is the largest current which may be drawn from the solar cell [25]. Since the short circuit current depends on the light generated carriers, calculations of the short circuit current can quantitatively account for optical losses across the entire spectral range. The short circuit current is calculated using AM1.5G data for solar spectrum, under the assumption that all photons transmitted into CdTe layer are completely absorbed.

If $\varnothing_{\mathrm{i}}$ is the spectral radiation power density and ' $h v$ ' is the photon energy, the spectral density of the incident photon flux will be $\emptyset_{\mathrm{i}} / \mathrm{hv}$. Thus, the short circuit current density (Figure 11) is given as:

$$
\mathrm{Jsc}=\sum_{i=300}^{850}\left(T(\lambda) * \emptyset_{\mathrm{i}}\left(\lambda_{\mathrm{i}}\right) * \Delta \lambda_{\mathrm{i}}\right) / \mathrm{hv}_{\mathrm{i}}
$$

where,

$\mathrm{Jsc}=$ Short circuit current

$\mathrm{T}(\lambda)=$ Total transmission

$\emptyset_{\mathrm{i}}=$ Spectral radiation density

$\mathrm{hv}_{\mathrm{i}}=$ Photon energy.

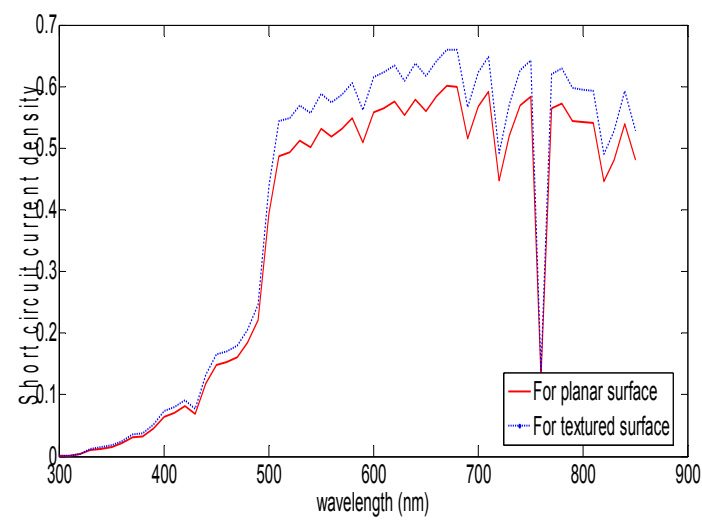

Figure 11. Effect of increase in Jsc due to light trapping.
It can be seen from the figure (8) that there will be more than $10 \%$ increase in short circuit current density of the CdTe solar cell deposited on textured surface compared to the conventional solar cell.

\section{CONCLUSION}

A feasibility study to manufacture a flexible and textured lightweight polymer substrate was carried out. Ordered wrinkles were obtained on the surface of PDMS polymer by treating it with Piranha solution and applying an external force. Theoretical calculations were performed, revealing an increase of more than $10 \%$ in radiation transmission and short circuit current density of the CdTe solar cell if a fabricated, textured substrate is used instead of a planar surface.

\section{ACKNOWLEDGEMENT}

Financial support provided by the National Science Foundation under Grant No CMMI-1400800 is acknowledged.

\section{REFERENCES}

1. Romeo, A., et al., High-efficiency flexible CdTe solar cells on polymer substrates. Solar Energy Materials and Solar Cells, 2006. 90(18-19): p. 3407-3415.

2. Mathew, X., et al., CdTe/CdS solar cells on flexible substrates. Solar Energy, 2004. 77(6): p. 831-838.

3. Seung-Bum, R., et al., An effective light trapping configuration for thin-film solar cells. Applied Physics Letters, 2007. 91(24): p. 243501243501-3.

4. Weidong, Z., et al., Microstructured surface design for omnidirectional antireflection coatings on solar cells. Journal of Applied Physics, 2007. 102(10): p. 103105-103105-9.

5. Shih, T.-K., et al., Topographic control on silicone surface using chemical oxidization method. Applied Surface Science, 2007. 253(24): p. 9381-9386.

6. Koh, K.-S., et al., Quantitative Studies on PDMS-PDMS Interface Bonding with Piranha Solution and its Swelling Effect. Micromachines, 2012. 3(2): p. 427-441.

7. Schweikart, A., et al., Controlled Wrinkling as a Novel Method for the Fabrication of Patterned Surfaces, in Complex Macromolecular Systems I, A.H.E. Müller and H.-W. Schmidt, Editors. 2010, Springer Berlin Heidelberg. p. 75-99.

8. Khang, D.-Y., et al., A Stretchable Form of Single-Crystal Silicon for High-Performance 
Electronics on Rubber Substrates. Science, 2006. 311(5758): p. 208-212.

9. Stafford, C.M., et al., A buckling-based metrology for measuring the elastic moduli of polymeric thin films. Nature materials, 2004. 3(8): p. 545-550.

10. Chung, S., et al., Non-Lithographic Wrinkle Nanochannels for Protein Preconcentration. Advanced Materials, 2008. 20(16): p. 30113016.

11. Yang, S., K. Khare, and P.-C. Lin, Harnessing Surface Wrinkle Patterns in Soft Matter. Advanced Functional Materials, 2010. 20(16): p. 2550-2564.

12. Moon, M.-W. and A. Vaziri, Surface modification of polymers using a multi-step plasma treatment. Scripta Materialia, 2009. 60(1): p. 44-47.

13. Kirill, E., et al., Nested self-similar wrinkling patterns in skins. Nature materials, 2005. 4(4): p. 293-297.

14. Moon, M.-W., et al., Wrinkled hard skins on polymers created by focused ion beam. Proceedings of the National Academy of Sciences, 2007. 104(4): p. 1130-1133.

15. Watanabe, M. and K. Mizukami, Well-Ordered Wrinkling Patterns on Chemically Oxidized Poly(dimethylsiloxane) Surfaces. Macromolecules, 2012. 45(17): p. 7128-7134.

16. Ned, B., et al., Spontaneous formation of ordered structures in thin films of metals supported on an elastomeric polymer. Nature, 1998. 393(6681): p. 146-149.

17. Gaewdang, T., N. Wongcharoen, and T. Wongcharoen, Characterisation of CdS/CdTe Heterojunction Solar Cells by Current-Voltage Measurements at Various Temperatures under Illumination. Energy Procedia, 2012. 15(0): p. 299-304.

18. Mohamed, H.A., Dependence of efficiency of thin-film CdS/CdTe solar cell on optical and recombination losses. Journal of Applied Physics, 2013. 113(9): p. 093105-093105-6.

19. Romeo, N., et al., Recent progress on $\mathrm{CdTe} / \mathrm{CdS}$ thin film solar cells. Solar Energy, 2004. 77(6): p. 795-801.

20. Engelbrecht, J.A.A., Modelling the reflectance of silicon. Infrared Physics \& Technology, 1994. 35(5): p. 701-708.

21. Adachi, S., Optical Constants of Crystalline and Amorphous Semiconductors, 1950, Kluwer academic publishers.

22. Reference Solar Spectral Irradiance [cited 2013 10/10/2013]; Available from: http://rredc.nrel.gov/solar/spectra/am1.5/.
23. U Gangopadhya, S.K.D., U Saha, Texturization and light trapping in silicon solar cells, 2009, Nova science publisher, Inc. p. 100.

24. Glassner, A.S., PRINCIPLES OF DIGITAL IMAGE SYNTHESIS. Vol. one. 1995, United states of America: Morgan Kaufmann Publishers, Inc.

25. Bowden, C.H.a.S. PVeducation.org. [cited 2013 10/10/2013]; Available from: http://pveducation.org/pvcdrom/solar-celloperation/short-circuit-current. 